\title{
Expanding the Perisurgical Home to Improve Postoperative Pain Management and Reduce Opioid Consumption
}

\author{
Ivan Urits, MD, ${ }^{1}$ Omar Viswanath, MD, ${ }^{2,3,4}$ Vwaire Orhurhu, MD, ${ }^{1}$ Viet Cai, MD, ${ }^{1}$ Musa Aner, MD ${ }^{1}$ \\ ${ }^{1}$ Department of Anesthesia, Critical Care and Pain Medicine, Harvard Medical School, Beth Israel Deaconess Medical Center, Boston, MA \\ ${ }^{2}$ Valley Anesthesiology and Pain Consultants, Phoenix, AZ ${ }^{3}$ Department of Anesthesiology, University of Arizona College of Medicine- \\ Phoenix, Phoenix, AZ ${ }^{4}$ Department of Anesthesiology, Creighton University School of Medicine, Omaha, NE
}

\section{TO THE EDITOR}

Following surgery, effective pain management is an important component of patient recovery and satisfaction. However, the conceptualization of pain as the fifth vital sign has led to a culture of drastic opioid overprescription. ${ }^{1,2}$ As pain scores and adequate pain management gained attention, the expectation that patients should experience no pain became widely accepted, along with the expectation that postoperative discharge must include an opioid prescription. Moreover, pain became an important factor in comparative analyses of hospitals, even contributing to physician reimbursement. As a result, physicians commonly feel pressured to prescribe an unnecessary amount of opioids to attempt to prevent inadequately controlled pain, emergency department visits, and hospital readmissions. Unsurprisingly, burdened with postoperative pain as a marker of quality, surgeons have become one of the highest prescribers of opioid medications. In the face of the resulting opioid epidemic, however, it is prudent for providers to revisit the topic of postoperative pain control and to reconsider the extent to which opioids play a role.

Given the expanding domain of the perisurgical home, anesthesiologists stand to play an integral role in improving postoperative patient pain management and opioid prescribing practices. The evaluation during a preanesthetic testing clinic appointment has become an important component of total anesthesia care of the patient. Typically, these clinic appointments include a review of the patient's medical history to ensure that appropriate testing and management of comorbid conditions have been done, so patients are optimized for their perisurgical passage. The preanesthetic testing appointment is a prime opportunity to provide education and to help guide patient expectations regarding postoperative pain, as well as to discuss strategies for how best to manage it. To help bridge perioperative patient care, anesthesia providers may make recommendations for appropriate postoperative pain management strategies for patients who may be less than straightforward and therefore challenging. In this way, anesthesiologists may reframe the paradigm of postoperative pain management and serve as the patient guardian throughout the perioperative passage to thus expand the role of the perisurgical home. Most important, improved communication among the preanesthetic clinician and the primary care and surgical teams can help improve patient analgesic management and minimize dependence on an opioid prescription for pain management.

Education about postoperative pain and its management can potentially reduce patient anxiety and prepare patients for reducing opioid analgesia. The preoperative discussion should involve an explanation of pain that includes the important information that the treatment of pain does not necessarily mean the absence of pain. The goal of pain management should be just that, to make the pain manageable. With this understanding, patients can be better readied to cope with the anticipated pain after surgery. During the preoperative discussion, the adverse effects of opioids should also be discussed in depth and the negatives of longterm opioid use explained. While opioid medication may be a necessary part of acute postoperative pain management, use should be minimized and every effort should be made to discontinue as soon as appropriate. As postoperative pain subsides, alternatives to opioid medications must be emphasized.

The anesthesia literature does not discuss how patient expectations of pain may influence their encounter with postoperative pain and subsequent requirement for opioid consumption. Similar models, however, have been shown to be effective in other fields. The orthopedic literature, for instance, has shown improvement in surgical outcomes in patients who have undergone preoperative education prior to elective hip or knee replacement. ${ }^{3}$ As such, a significant aspect of treatment success may be attributed to the expectations patients have regarding their postoperative experience. Applying a similar concept to the management of postoperative pain would be interesting. Studies assessing the impact of preoperative education about pain and opioid therapy on postoperative opioid consumption and patient satisfaction are needed.

Finally, increased attention must be given to standardization of postoperative pain management and opioid prescribing. The need for consensus on recommendations and guidelines is evidenced by the highly variable opioid prescribing practices across providers. Widely differing postoperative opioid prescriptions are provided by different 
providers to similar patients undergoing similar procedures. ${ }^{4}$ Moreover, while guidelines for opioid management in the treatment of chronic pain have significantly impacted opioid prescribing practices, clinical practice guidelines for the management of postoperative pain have not gained traction. ${ }^{5,6}$ Although creating a one-size-fits-all formula that would encompass individual patient pain management requirements for various surgeries would be difficult, interspecialty collaboration among surgeons, anesthesiologists, and primary care physicians can help address the absence of opioid prescription guidelines for acute postoperative pain.

\section{REFERENCES}

1. Geriatrics and Extended Care Strategic Healthcare Group National Pain Management Coordinating Committee Veterans Health Administration. Pain as the 5th vital sign toolkit. Department of Veterans Affairs. www.va.gov/ PAINMANAGEMENT/docs/Pain_As_the_5th_Vital_Sign_ Toolkit.pdf. Published October 2000. Accessed April 11, 2019.
2. Quality improvement guidelines for the treatment of acute pain and cancer pain. American Pain Society Quality of Care Committee. JAMA. 1995 Dec 20;274(23):1874-1880.

3. McDonald S, Page MJ, Beringer K, Wasiak J, Sprowson A. Preoperative education for hip or knee replacement. Cochrane Database Syst Rev. 2014 May 13;(5):CD003526. doi: 10.1002/14651858.CD003526.pub3.

4. Hill MV, McMahon ML, Stucke RS, Barth RJ Jr. Wide variation and excessive dosage of opioid prescriptions for common general surgical procedures. Ann Surg. 2017 Apr;265(4):709-714. doi: 10.1097/SLA.0000000000001993.

5. Dowell D, Haegerich TM, Chou R. CDC guideline for prescribing opioids for chronic pain - United States, 2016. MMWR Recomm Rep. 2016 Mar 18;65(1):1-49. doi: 10.15585/mmwr.rr6501e1.

6. Chou R, Gordon DB, de Leon-Casasola OA, et al. Management of postoperative pain: a clinical practice guideline from the American Pain Society, the American Society of Regional Anesthesia and Pain Medicine, and the American Society of Anesthesiologists' Committee on Regional Anesthesia, Executive Committee, and Administrative Council. J Pain. 2016 Feb;17(2):131-157. doi: 10.1016/j.jpain.2015.12.008. 